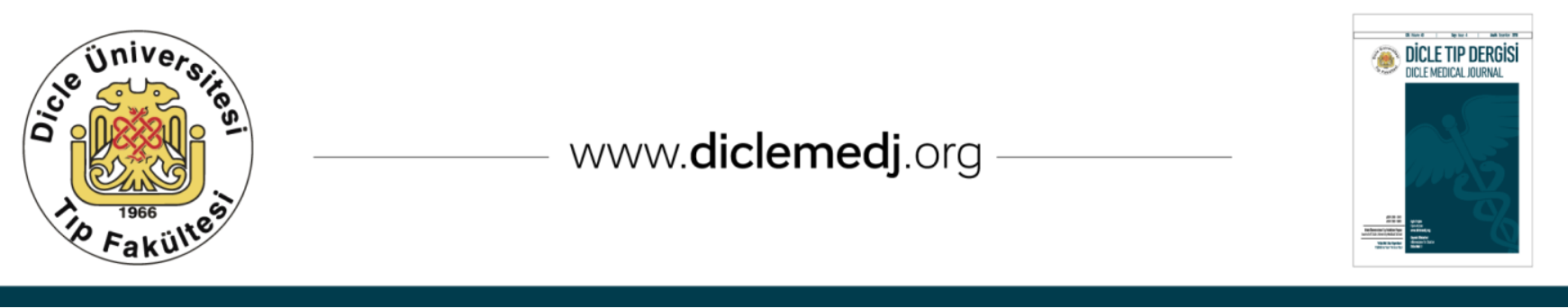

Özgün Araştırma / Original Article

\title{
Görsel Uyaranlara İliş̧kin Manyetoensefalografi Sinyallerinin Genelleştirilmiş Regresyon Sinir Ağı ile Sınıflandırılması
}

\author{
Onursal Çetin'1, Feyzullah Temurtaş² \\ 1 Bandırma Onyedi Eylül Üniversitesi, Mühendislik ve Doğa Bilimleri Fakültesi, Elektronik ve Haberleşme Müh. Böl., 10200, Bandırma, Balıkesir, Türkiye \\ ORCID: 0000-0001-5220-3959 \\ 2 Bandırma Onyedi Eylül Üniversitesi, Mühendislik ve Doğa Bilimleri Fakültesi, Elektronik ve Haberleşme Müh. Böl., 10200, Bandırma, Balıkesir, Türkiye \\ ORCID: 0000-0002-3158-4032
}

Geliş: 13.07.2018; Revizyon: 10.09.2018; Kabul Tarihi: 26.11.2019

Öz

Amaç: Bu çalışmanın amacı, beyin aktivitesini çözmek için Manyetoensefalografi (MEG) sinyallerini yapay sinir ağı ile sinıflandırmaktır.

Yöntemler: MEG sinyallerini sınıflandırmak için Genelleștirilmiş Regresyon Sinir Ağı (GRSA) kullanılmıștır. Riemannian yaklaşımı ile sinyallerin öznitelikleri çıkarılmış ve 10 katlı çapraz doğrulama tekniği ile GRSA'nın doğruluğu hesaplanmıştır.

Bulgular: Çalışmada 9 kız, 7 erkek bireye ait 306 kanaldan kaydedilen MEG verileri kullanılmıştır. Her bireye yaklaşık 588 uyaran gösterilmiştir ve böylece tüm veri seti 9414 uyarandan oluşmaktadır. Ortalama spesifite, ortalama duyarlılık ve ortalama sınıflandırma doğruluğu sırasıyla \%75,43, \%82,57 ve \%79 olarak elde edilmiştir. Bu çalışma ve aynı MEG veri setini kullanan diğer çalışmalar tarafından elde edilen sınıflandırma doğrulukları karşılaştırmalı olarak sunulmuştur.

Sonuç: GRSA'nın MEG sinyallerinin sınıflandırılmasında kullanılan mevcut yöntemlere başarılı bir alternatif oluşturduğu düşünülmektedir.

Anahtar kelimeler: Manyetoensefalografi Genelleștirilmiş regresyon sinir ağı, Sınıflandırma, Riemannian yaklaşımı.

DOI: $10.5798 /$ dicletip

Yazışma Adresi / Correspondence: Onursal Çetin, Bandırma Onyedi Eylül Üniversitesi, Mühendislik ve Doğa Bilimleri Fakültesi, Elektronik ve Haberleșme Mühendisliği Bölümü, 10200, Bandırma, Balıkesir, Türkiye e-mail: ocetin@bandirma.edu.tr 


\title{
Classification of Magnetoencephalography Signals Regarding Visual Stimuli by Generalized Regression Neural Network
}

\begin{abstract}
Objective: The aim of this study is to classify the magnetoencephalography (MEG) signals with artificial neural network to solve brain activity.

Methods: The Generalized Regression Neural Network (GRNN) was used to classify MEG signals. The features of the signals were extracted by the Riemannian approach and the accuracy of the GRNN was calculated by the 10 -fold cross validation technique.

Results: In the study, MEG data recorded from 306 channels belonging to 7 male subjects and 9 female subjects were used. Approximately 588 stimuli were shown to each individual, so the entire data set is composed of 9414 stimuli. Mean specificity, mean sensitivity and mean classification accuracy were obtained 75.43\%, 82.57\% and 79\%, respectively. The classification accuracies obtained by this study and other studies for same MEG dataset were presented comparatively.
\end{abstract}

Conclusion: GRNN is thought to be a successful alternative to existing methods for classifying MEG signals.

Keywords: Magnetoencephalography, Generalized regression neural network, Classification, Riemannian approach.

\section{GíRiş}

Beyin-bilgisayar arayüzü (BBA) zihinsel aktivite ile insan-makine haberleşmesini olanaklı hale getiren sistemdir. Sinir hücrelerinin elektrokimyasal etkileşimi sonucu ortaya çıkan beyin sinyallerinin kaydedilmesi, bu sistemler için en temel seviyedir ${ }^{1}$. BBA sistemleri, çeşitli tekniklerle alınan beyin sinyallerinin işlenmesi ile gerçekleștirilmektedir. Manyetoensefalografi (MEG), BBA sistemleri gerçekleştirmek için zihinsel aktivitenin tespitinde kullanılan yöntemlerden biridir. MEG, nöronal aktivite tarafından üretilen küçük manyetik alanların çoklu ölçümlerini her milisaniyede gerçekleştiren ve invazif olmayan fonksiyonel görüntüleme tekniğidir².

İnsan beyni milisaniyeler mertebesinde görsel algılama hızına sahiptir ve bu mükemmel görsel sistematiğin nasıl çalıştı̆̆ını anlamak nörobilim araştırmaları açısından önemlidir ${ }^{3}$. Bireye bir uyaran gösterildiğinde, uyaran ve beyinde oluşan sinyal arasındaki ilişki, makine öğrenmesi algoritmaları geliştirmek için kullanılır. MEG sinyalleri kullanılarak, eş zamanlı beyin aktivitesinden bireye sunulan uyaranın tahmin edilmesinde, Yapay Sinir Ağları (YSA) kullanılarak geliştirilen sinıflandırıcılar kullanılabilirler"

YSA, insan beyninin sistematiğinden esinlenen ve sınıflandırma çalışmalarında sıklıkla kullanılan bilgisayar sistemleridir ${ }^{5}$. Sınıflandırma işlemi gerçekleştirilirken sinıflandırıcıyı eğitmek ve sinıflandırıcı doğruluğunu test etmek gerekmektedir. MEG sinyallerinin karmaşıklığı, çevresel değişkenler ve beynin yapısından kaynaklanan fonksiyonel farklılıklar sinıflandırma işlemini zorlaștırmaktadır ${ }^{4}$. MEG görüntüleme tekniğinin kolay ulaşılamayan yapısı sebebiyle bu tekniğin kullanıldığı çalışmalar sınırlı sayıdadır ${ }^{4,6-12}$. Sinıflandırıcının performansını artırmak için hem iyi bir sinıflandırma algoritmasına hem de başarılı bir öznitelik çıkarım yöntemine ihtiyaç duyulmaktadır ${ }^{4}$. Literatürde çok kanallı ve karmaşık yapıdaki beyin sinyallerinin özniteliklerinin çıkarılmasında çeşitli yöntemler kullanılmıştır ${ }^{13,15}$. Öznitelik çıkarım yöntemleri arasında Riemannian yaklaşımı rekabetçi özelliğiyle öne çıkmaktadır ${ }^{15,16}$. 
$\mathrm{Bu}$ çalışma kapsamında, Specht tarafından önerilen Genelleştirilmiş Regresyon Sinir Ağ (GRSA) kullanılarak MATLAB (Lisans no: 40692431) programı vasıtasıyla MEG sinyalleri sinıflandırılmıştır. $\mathrm{Bu}$ amaçla, Henson ve arkadaşları tarafından sunulan MEG veri setinden faydalanılmıştır ${ }^{17}$. Öncelikle karmaşık ve çok kanallı MEG sinyali filtrelenmiş ve gürültüden arındırılmıştır. Daha sonra Riemannian yaklaşımı ${ }^{15,16}$ kullanılarak öznitelik çıkarım işlemi gerçekleştirilmiş, çok kanallı MEG sinyallerinin boyutu azaltılmıştır. 10 katlı çapraz doğrulama tekniği GRSA ağının doğruluğunu hesaplamak için kullanılmıştır ${ }^{18}$. Elde edilen sonuçlar, aynı MEG veri setini kullanan önceki yöntemler ile karşılaştırılmıştır.

\section{YÖNTEMLER}

\section{Manyetoensefalografi Veri Seti}

“Dec2Meg2014"19 yarışması kapsamında yeniden düzenlenen MEG veri seti Henson ve arkadaşları tarafından sunulmuștur ${ }^{17}$. Bu yarışmada amaç, bireye sunulan görsel uyaranın sınıfının beyin aktivitesinden tahmin edilmesidir. 9'u kız, 7'si erkek toplam 16 bireye sıradan kişilerin yüzleri, ünlü kişilerin yüzleri ve bu resimlerin anlamsız halleri olmak üzere 3 farklı uyaran gösterilmiş ve 306 kanaldan MEG sinyalleri kaydedilmiştir.

$\mathrm{Bu}$ çalışmada, MEG veri seti Olivetti ve arkadaşlarının düzenledikleri şekliyle kullanılmıştır. Yapılan düzenlemeyle resimler normal yüz ve anlamsız yüz olmak üzere iki sınıfa ayrılmıștır ${ }^{2}$. Veri seti hakkında daha detaylı bilgi [19] numaralı referanstan edinilebilir. Normal yüz ve anlamsız yüz örnek resimleri Şekil 1'de gösterilmiştir ${ }^{19}$.

Normal yüz ya da anlamsız yüzlerden oluşan yaklaşı 588 resim, 16 bireye rastgele ve seri şekilde 1 saniye boyunca gösterilmiş ve bireyler her bir resimden sonra 0,5 saniye dinlendirilmiștir. 16 bireye toplamda 9414 resim gösterilmiştir.
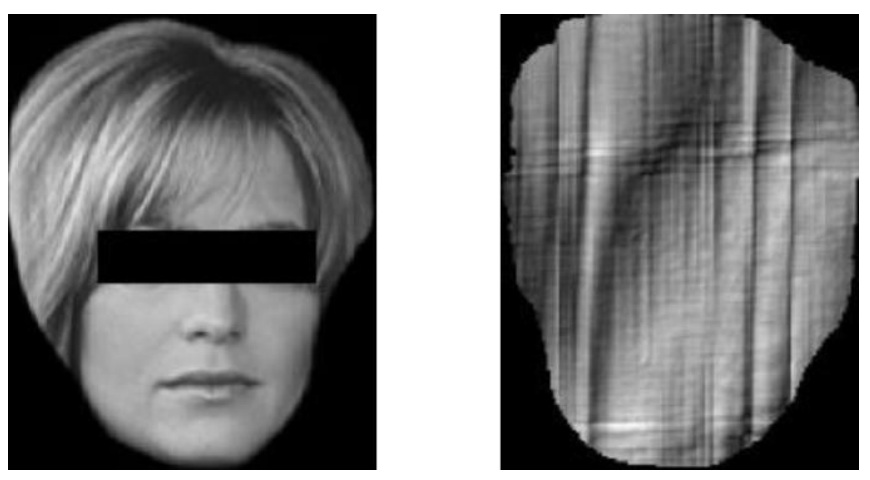

Şekil 1. Normal yüz ve anlamsız yüz örnekleri

\section{Özniteliklerin Belirlenmesi}

MEG verileri GRSA'ya sunulmadan önce sinyallerin örnekleme frekansı $250 \mathrm{~Hz}$ 'e düşürülmüş ve bant geçiren filtre yardımıyla gürültüden arındırılmıştır². Filtreleme işlemi için 10. Dereceden Butterworth 1-20 Hz bant geçiren filtre kullanılmıştır. MEG sinyalleri çok kanallı olup hangi kanalların kritik bilgi içerdiğinin belirlenmesi önemlidir ${ }^{3}$. Bu nedenle öznitelik çıkarma işleminin önemi büyüktür. Konumsal filtreleme yardımıyla 306 olan kanal sayısı 8'e düşürülmüștür. Daha sonra bu kanallardan alınan sinyallerden elde edilen kovaryans matrislerinin arasındaki Riemannian mesafesi ${ }^{20,21}$ hesaplanarak öznitelikler elde edilmiştir22.

\section{Genelleştirilmiş Regresyon Sinir Ă̆ı ile MEG Sinyallerinin Sinıflandırılması}

Genelleştirilmiş regresyon sinir ağl, hedef değişkenler sürekli olduğunda doğrusal olmayan regresyonlar gerçekleştir. $\mathrm{Bu}$ regresyon analizi, veri davranışını taklit eden fonksiyonel formun tahmin edilmesini gerektirir. Çok boyutlu bir problemde, lineer olmayan regresyon analizi ile bu fonksiyonu seçmek zordur. Donald Specht tarafından 1991 yılında önerilen GRSA algoritması, varsayılan bir işlevsel forma ihtiyaç duymadığından bu dezavantajı başarılı bir şekilde aşmaktadır ${ }^{23}$. Geriye yayılım metodundan farklı olarak iteratif bir eğitim süreci gerektirmeyen GRSA, radyal tabanlı çalışan ileri beslemeli bir YSA 
modelidir24. 'F' giriş vektörü için GRSA çıkışı ' $Y$ ' şu şekilde hesaplanır;

$$
Y(F)=\frac{\sum_{i=1}^{n} W_{i} \exp \left[-\frac{D_{i}^{2}}{2 \sigma^{2}}\right]}{\sum_{i=1}^{n} \exp \left[-\frac{D_{i}^{2}}{2 \sigma^{2}}\right]}
$$

$\mathrm{Bu}$ denklemde, $n$ gözlenen örnek sayısını; $\mathrm{D}_{i}{ }^{2}=\left(F-F_{i}\right)^{\mathrm{T}} \quad\left(F-F_{i}\right)$ mesafe fonksiyonunu; $F_{i}$ örnek değerlerini ve $W$ i ağırlık değerlerini; $\sigma$ ise yayllım parametresini ifade etmektedir. MEG sinyalinin sınıflandırılması için kullanılan GRSA yapısı Şekil 2'de verilmiştir.

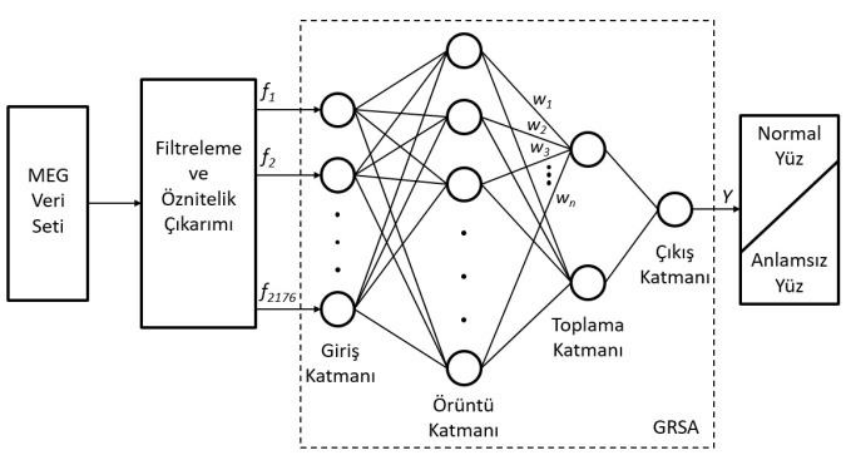

Şekil 2. Sınıflandırmada kullanılan GRSA yapısının şematik gösterimi

GRSA, her biri bir sonraki katmanla bağlantılı olmak üzere giriş katmanı, örüntü katmanı, toplama katmanı ve çıkış katmanı olmak üzere dört katmandan oluşmaktadır. $\mathrm{Bu}$ dört katmandan her biri farklı sayılarda nörona sahiptir. Giriş katmanındaki nöronların sayısı bağımsız değişkenlerin sayısına eşittir. Eğitim veri setindeki her örnek veri için örüntü katmanında bir adet nöron ilişkilendirilmesi sebebiyle işlem sayısı artmaktadır. Eğitim esnasında, giriş vektörünün her bir giriş nöronu için Öklid mesafesi hesaplanır. Her bir giriş nöronundan gelen mesafeler, doğrusal olmayan üstel bir aktivasyon fonksiyonuna bir düzeltme parametresi ile beslenir. Ortaya çıkan değerler toplama katmanına aktarılır. Toplama katmanı, pay ve payda adı verilen iki nöron içerir. Payda nöronu, örüntü katmanının çıktılarının toplamını hesaplar. Pay nöronu ise örüntü katmanı çıtıları ile her eğitim verisinin beklenen değerlerini içeren bir vektör arasındaki nokta çarpımı hesaplar. Çıkış katmanında pay nöronundan gelen değer, payda nöronundan gelen değere bölünerek tahmini değer bulunur ${ }^{23,24}$.

\section{Sınıflandırma Doğruluğu}

Sistem performansını belirlemek için performans değerlendirme ölçütlerinden faydalanılmaktadır. Bu çalışmada Denklem 2, Denklem 3 ve Denklem 4 ile verilen sınıflandırma doğruluğunun yanı sıra Denklem 5 ile verilen spesifite ve Denklem 6 ile verilen duyarlılık değerlendirme ölçütleri de kullanılmıştır ${ }^{25,26 .}$

$$
\begin{aligned}
& \operatorname{Doğruluk}(N)=\frac{\sum_{i=1}^{|N|} \operatorname{Tahmin}\left(n_{i}\right)}{|N|}, n_{i} \in N \\
& \operatorname{Tahmin}(n)=\left\{\begin{array}{l}
1, \text { Ĕger Tahmin }(n)=n c \\
0, \text { aksi halde }
\end{array}\right.
\end{aligned}
$$

Sınıflandırma Doğruluğu (Alg)

$$
=\frac{\sum_{i=1}^{|k|} \text { Doğruluk }\left(N_{i}\right)}{|k|}
$$

Burada; $N$, sinıflandırılan (test) veri setini, $n c, n$ değerinin sınıfını, Tahmin (n), n'nin sınıflandırma sonucunu ve $k$ değeri de k-katlı çapraz doğrulama parametresini ifade etmektedir. Spesifite ve duyarlılık şu şekilde hesaplanmaktadır;

$$
\begin{aligned}
& \text { Spesifite }=\frac{T N}{T N+F P} \\
& \text { Duyarlılık }=\frac{T P}{T P+F N}
\end{aligned}
$$

Burada;

- Yanlış pozitif (FP): Anlamsız yüzlerin, normal yüz olarak sınıflandırma karar sayısı.

- Yanlış negatif (FN): Normal yüzlerin, anlamsız yüz olarak sınıflandırma karar sayısı.

- Gerçek pozitif (TP): Normal yüzlerin, normal yüz olarak sınıflandırma karar sayısı. 
- Gerçek negatif (TN): Anlamsız yüzlerin, anlamsız yüz olarak sınıflandırma karar sayısı.

\section{K-katlı Çapraz Doğrulama}

Sistem doğruluğunun sınanması için veri kümesi, eğitim kümesi ve test kümesi olmak üzere iki parçaya ayrılmaktadır. Çalışma içerisinde GRSA ağının doğruluğu hesaplanırken K-katlı çapraz doğrulama tekniği kullanılmıştır. ' $\mathrm{k}$ ' parametresi, veri setinin eșit büyüklükte kaç alt kümeye bölüneceğini belirtmektedir. Veri seti sinıflara ayrılır ve sonrasında sinıflandırma algoritması k-kez eğitilir ve test edilir. Her defasında $\mathrm{k}$ adet kümeden bir tanesi test işlemi için ayrılmakta ve geriye kalan k-1 küme sınıflandırıcının eğitimi için kullanılmaktadır. Böylece, $\mathrm{k}$ adım sonunda elde edilen k-farklı test sonucunun ortalaması alınarak genel sinıflandırma doğruluğu elde edilmektedir ${ }^{27}$. Bu çalışmada GRSA ağının doğruluğunu hesaplamak amacıyla 10-katlı çapraz doğrulama tekniği kullanılmıştır ${ }^{18}$.

\section{SONUÇLAR}

$\mathrm{Bu}$ çalışmada, 16 bireye ait 306 kanaldan kaydedilen MEG verileri kullanılmıştır. Her bir bireye yaklaşık 588 uyaran olmak üzere toplam 9414 uyaran gösterilmiştir. Gürültü içeren MEG sinyalleri filtrelenip gürültüden arındırılmış ve öznitelikleri elde edilmiştir. 2176 özniteliğe sahip 9414 uyaran rastgele GRSA ağına sunulmuştur. Çalışmanın amacl, GRSA kullanılarak MEG sinyalinin "normal yüz" ya da "anlamsız yüz" sınıflarından hangisine ait olduğunun belirlenmesidir.

10-katlı çapraz doğrulama tekniği ile sınıflandırıcının doğruluğu sınanmıştır. Bu amaçla veri seti 10 eşit parçaya (kat) bölünmüştür. GRSA toplamda 10 kere eğitilip test edilmiş, her defasında parçalardan biri test işlemine ayrilıp geriye kalan 9 parça ağın eğitimi için kullanılmıştır. 10 parça için elde edilen doğrulukların ortalaması alınarak sınıflandırma doğruluğu elde edilmiştir. Her bir parça için düzeltme parametreleri ve hesaplanan duyarlılık, spesifite ve doğruluk ile bu parametrelere ait ortalama başarımlar Tablo 1'de yer almaktadır.

Tablo 1: 10-katlı çapraz doğrulama için başarım oranları

\begin{tabular}{|c|c|c|c|c|}
\hline & \multirow{2}{*}{$\begin{array}{c}\text { Yayılım } \\
\text { Parametresi } \\
(\sigma)\end{array}$} & \multicolumn{3}{|c|}{ Spesifite Duyarlılık Doğruluk } \\
\hline & & $(\%)$ & $(\%)$ & $(\%)$ \\
\hline Kat 1 & 1.6367 & 75,71 & 82,98 & 79,35 \\
\hline Kat 2 & 1.3924 & 74,71 & 82,24 & 78,48 \\
\hline Kat 3 & 1.3185 & 75,24 & 82,36 & 78,80 \\
\hline Kat 4 & 1.4961 & 75,11 & 82,56 & 78,84 \\
\hline Kat 5 & 1.6155 & 75,54 & 82,36 & 78,95 \\
\hline Kat 6 & 0.5356 & 75,51 & 82,33 & 78,92 \\
\hline Kat 7 & 1.7698 & 75,35 & 82,53 & 78,94 \\
\hline Kat 8 & 1.5768 & 75,66 & 82,66 & 79,16 \\
\hline Kat 9 & 1.4128 & 75,73 & 82,75 & 79,24 \\
\hline Kat 10 & 1.6418 & 75,74 & 82,91 & 79,33 \\
\hline ORTALAMA & & 75,43 & 82,57 & 79,00 \\
\hline
\end{tabular}

Tablo 1 incelendiğinde, katlar arası veri dağılımının birbirini dışlayan bir yapıda rastgele olarak gerçekleştiği ve sonuç olarak katlar arası doğruluğun birbirine yakın olduğu gözlenmektedir. Aynı zamanda duyarlılığın yüksek olması normal yüzün anlamsız yüze göre daha iyi ayırt edildiği anlamına gelmektedir. Ortalama spesifite, ortalama duyarlılık ve ortalama sınıflandırma doğruluğu sirasiyla $\% 75,43, \% 82,57$ ve $\% 79$ olarak elde edilmiştir.

Aynı MEG verisini kullanan yöntemler ve bu çalışma tarafından elde edilen sınıflandırma doğrulukları karşılaștırmalı olarak Tablo 2'de gösterilmiştir.

Tablo 2'den de görüldüğü gibi Abdullah Çalışkan ve arkadaşları kullandıkları algoritmalar ile \%68,36 ile \%80,85 arasında değisşen sınıflandırma doğrulukları elde etmiş̧lerdir ${ }^{3}$. En iyi performansı Derin Sinir Ağı (Deep Neural Network-DNN) gösterirken en 
düşük performansı Karar Ağacı (Decision Trees-DT) yapısı göstermiştir. Çetin ve Temurtaş yaptıkları çalışmada öğrenmeli vektör kuantalama (Learning Vector Quantization-LVQ) kullanmışlar ve \%69,39 sınıflandırma doğruluğu elde etmişlerdir ${ }^{4}$. Bu çalışma ise en iyi sonucu göstermese de GRSA'nın performans açısından \%79 ile Destek Vektör Makinesi (Support Vector Machine SVM) yapısından daha iyi olduğu ve DNN yapısına yakın sonuç verdiği görülmektedir.

Tablo 2: MEG verisi için kullanan yöntemler ve erişilen sınıflandırma doğrulukları

\begin{tabular}{ccc}
\hline Çalışmalar & $\begin{array}{c}\text { Uygulanan } \\
\text { Yöntemler }\end{array}$ & $\begin{array}{c}\text { Sınıflandırma } \\
\text { Doğrulukları (\%) }\end{array}$ \\
\hline & DNN & 80.85 \\
& SVM & 78.01 \\
{$[3]$} & KNN & 72.84 \\
& NB & 71.92 \\
& DT & 68.36 \\
\hline [4] & LVQ & 69.39 \\
\hline Bu çalışma & GRSA & $\mathbf{7 9 . 0 0}$ \\
\hline
\end{tabular}

TARTIŞMA

MEG sinyalleri çok kanallı ve gürültü içeren yapıda olup bu sinyallerden anlaml bilgi edinilmesi bazı işlemler gerektirmektedir. $\mathrm{Bu}$ çalışma kapsamında, MEG sinyallerinin sınıflandırılmasında daha önce başarımı incelenmemiş bir yöntem olan GRSA kullanılmıştır. $\mathrm{Bu}$ amaçla GRSA algoritması MATLAB programı üzerinde çalıştırılmıştır. 10katlı çapraz doğrulama tekniği ile sınıflandırıcının doğruluğu sınanmış, performans değerlendirme ölçütleri olan spesifite ve duyarlılık da hesaplanmıştır. GRSA, literatürde sınıflandırma işlemi için sıklıkla kullanılan SVM ve $\mathrm{K}$ En Yakın Komşu (KNearest Neighbours-KNN) yöntemleri ile kıyaslandığında daha başarılı ve kullanımı gün geçtikçe artan fakat gerçekleştirilmesi yazılım kütüphanelerinin yaygın olmaması nedeniyle uzmanlık gerektiren DNN yapısına yakın sonuç vermiştir. Buradan yola çıarak, GRSA'nın MEG sinyallerinin sinıflandırılmasında kullanılan mevcut yöntemler için başarılı bir alternatif oluşturduğu düşünülmektedir.

Çıkar Çatışması Beyanı: Yazarlar çıkar çatışması olmadığını bildirmişlerdir.

Finansal Destek: Bu çalışma her hangi bir fon tarafından desteklenmemiştir.

Declaration of Conflicting Interests: The authors declare that they have no conflict of interest.

Financial Disclosure: No financial support was received.

\section{KAYNAKLAR}

1. Bascil MS, Tesneli AY, Temurtas F. A study on analog and digital EEG signal filtering for brain computer interfaces (BCI). Electronic Letters on Science\&Engineering 2014; 10: 1-10.

2. Olivetti E, Kia SM, Avesani P. MEG decoding across subjects. International Workshop on Pattern Recognition in Neuroimaging 2014; doi: 10.1109/PRNI.2014.6858538.

3. Caliskan A, Yuksel ME, Badem H, Basturk A. A deep neural network classifier for decoding human brain activity based on Magnetoencephalography. Elektronika ir Elektrotechnika 2017; 23: 63-7.

4. Cetin 0 , Temurtas F. Öğrenmeli vektör kuantalama ile beyin bilgisayar arayüzü üzerine bir çalışma. Sakarya University Journal of Computer and Information Sciences 2018; 1: 17.

5. Gulbag A, Temurtas F. A study on quantitative classification of binary gas mixture using neural networks and adaptive neuro-fuzzy inference systems. Sensors and Actuators B 2006; 115: 252-62.

6. Abadi MK, Subramanian R, Kia SM, Avesani P, Patras I, Sebe N. DECAF: MEG-based multimodal database for decoding affective physiological responses. IEEE Trans. Affective Computing 2015; 6: 209-22.

7. Chan AM, Halgren E, Marinkovic K, Cash SS. Decoding word and category-specific spatiotemporal representations from MEG and EEG. NeuroImage 2011; 54: 3028-39.

8. Daliri MR. A hybrid method for the decoding of spatial attention using the MEG brain signals. Biomedical Signal Processing and Control 2014; 10: 308-12. 
9. Kia SM, Pedregosa F, Blumenthal A, Passerini A. Grouplevel spatio-temporal pattern recovery in MEG decoding using multi-task joint feature learning. Journal of Neuroscience Methods 2017; 285: 97-108.

10. Huttunen H, Kauppi JP, Tohka J. Regularized logistic regression for mind reading with parallel validation. ICANN 2011 International Conference on Artificial Neural Networks, Pattern Analysis, Statistical Modelling and Computational Learning2 MEG MindReading 2011; 20-24.

11. Kia SM, Vega Pons S, Weisz N, Passerini A. Interpretability of multivariate brain maps in linear brain decoding: Definition, and heuristic quantification in multivariate analysis of MEG time-locked effects. Frontiers in Neuroscience 2017; 10: 619; doi: 10.3389/fnins.2016.00619

12. Redcay E, Carlson TA. Rapid neural discrimination of communicative gestures. Social Cognitive and Affective Neuroscience 2015; 10: 545-51.

13. Ursulean R, Lazar AM. Detrended cross-correlation analysis of biometric signals used in a new authentication method. Elektronika ir Elektrotechnika 2009; 89: 55-8.

14. Raudonis V, Narvydas G, Simutis R. A classification of flash evoked potentials based on artificial neural network. Elektronika ir Elektrotechnika 2008; 81: 31-6.

15. Barachant A, Bonnet S, Congedo M, Jutten C. Multiclass brain-computer interface classification by Riemannian geometry. IEEE Trans. Biomedical Engineering 2012; 59: 920-28.

16. Yger F, Berar M, Lotte F. Riemannian approaches in brain computer interfaces: a review. IEEE Trans. Neural Systems and Rehabilitation Engineering 2017; 25: 1753-62.

17. Henson RN, Wakeman DG, Litvak V, Friston KJ. A parametric empirical Bayesian framework for the EEG/MEG inverse problem: generative models for multi-subject and multi-modal integration. Frontiers in Human Neuroscience 2011; 5: 76.
18. Cetin 0, Temurtas F, Gulgonul S. An application of multilayer neural network on hepatitis disease diagnosis using approximations of sigmoid activation function. Dicle Medical Journal 2015; 42: 150-7.

19. Ulaşabileceği adres:

https://www.kaggle.com/c/decoding-the-human-brain 20. Moakher M. A differential geometric approach to the geometric mean of symmetric positive-definite matrices. SIAM Journal on Matrix Analysis and Applications 2005; 26: 735-47.

21. Ulaşabileceği adres: https://github.com/alexandrebarachant/covariancetoolbox

22. Pennec $X$, Fillard $P$, Ayache N. A Riemannian framework for tensor computing. International Journal of Computer Vision 2006; 66: 41-66.

23. Specht DF. A general regression neural network. IEEE Transactions on Neural Networks 1991; 2: 568-76.

24. Specht DF, Shapiro PD. Generalization accuracy of probabilistic neural networks compared with backpropagation networks. IJCNN-91-Seattle International Joint Conference on Neural Networks 1991;887-92; doi: 10.1109/IJCNN.1991.155296.

25. Bascil MS, Cetin O, Er O, Temurtas F. Olasılıksal sinir ağının (PNN) parkinson hastalığının teşhisinde kullanilması. Electronic Letters on Science \& Engineering 2012; 8: 1-10.

26. Gorur K, Bozkurt MR, Bascil MS, Temurtas F. Glossokinetic potential based tongue-machine interface for 1-D extraction. Australas Phys Eng Sci Med 2018; 41: 379-91.

27. Bascil MS, Oztekin H. A study on hepatitis disease diagnosis using probabilistic neural network. Journal of Medical Systems 2012; 36: 1603-6. 